OPEN ACCESS

Edited by:

Ken Yokawa,

University of Bonn, Germany

Reviewed by:

Nabil I. Elsheery,

Tanta University, Egypt

Roy Navarre,

United States Department of

Agriculture, USA

*Correspondence:

Guozhang Kang,

The National Engineering Research Centre for Wheat, Henan Agricultural University, Zhengzhou, \#62, Nongye Road, 450002 Henan Province, China zkangg@163.com

Specialty section: This article was submitted to

Plant Physiology, a section of the journal Frontiers in Plant Science

Received: 06 February 2015 Accepted: 08 June 2015 Published: 30 June 2015

Citation:

Wei L, Wang L, Yang Y, Wang P, Guo $T$ and Kang $G$ (2015) Abscisic acid

enhances tolerance of wheat seedlings to drought and regulates transcript levels of genes encoding ascorbate-glutathione biosynthesis.

Front. Plant Sci. 6:458

doi: 10.3389/fpls.2015.00458

\section{Abscisic acid enhances tolerance of wheat seedlings to drought and regulates transcript levels of genes encoding ascorbate-glutathione biosynthesis}

\author{
Liting Wei ${ }^{1,2}$, Lina Wang ${ }^{2}$, Yang Yang ${ }^{2}$, Pengfei Wang ${ }^{1}$, Tiancai Guo ${ }^{1}$ and \\ Guozhang Kang ${ }^{1,3 *}$
}

\begin{abstract}
${ }^{1}$ The Collaborative Innovation Center of Henan Food Crops, College of Agronomy, Henan Agricultural University, Zhengzhou, China, ${ }^{2}$ The National Key Laboratory of Wheat and Maize Crop Science, College of Agronomy, Henan Agricultural University, Zhengzhou, China, ${ }^{3}$ The National Engineering Research Centre for Wheat, Henan Agricultural University, Zhengzhou, China
\end{abstract}

Glutathione (GSH) and ascorbate (ASA) are associated with the abscisic acid (ABA)-induced abiotic tolerance in higher plant, however, its molecular mechanism remains obscure. In this study, exogenous application $(10 \mu \mathrm{M})$ of ABA significantly increased the tolerance of seedlings of common wheat (Triticum aestivum L.) suffering from 5 days of $15 \%$ polyethylene glycol (PEG)-stimulated drought stress, as demonstrated by increased shoot lengths and shoot and root dry weights, while showing decreased content of hydrogen peroxide $\left(\mathrm{H}_{2} \mathrm{O}_{2}\right)$ and malondialdehyde (MDA). Under drought stress conditions, ABA markedly increased content of GSH and ASA in both leaves and roots of ABA-treated plants. Temporal and spatial expression patterns of eight genes encoding ASA and GSH synthesis-related enzymes were measured using quantitative real-time reverse transcription polymerase chain reaction (qPCR). The results showed that ABA temporally regulated the transcript levels of genes encoding ASA-GSH cycle enzymes. Moreover, these genes exhibited differential expression patterns between the root and leaf organs of ABA-treated wheat seedlings during drought stress. These results implied that exogenous ABA increased the levels of GSH and ASA in drought-stressed wheat seedlings in time- and organ-specific manners. Moreover, the transcriptional profiles of ASA-GSH synthesis-related enzyme genes in the leaf tissue were compared between ABA- and salicylic acid (SA)-treated wheat seedlings under PEG-stimulated drought stress, suggesting that they increased the content of ASA and GSH by differentially regulating expression levels of ASA-GSH synthesis enzyme genes. Our results increase our understanding of the molecular mechanism of ABA-induced drought tolerance in higher plants.

Keywords: abscisic acid, ascorbate, drought stress, glutathione, transcription level, Triticum aestivum L. 


\section{Introduction}

It is known that biotic and abiotic stresses (including drought) induces the generation of reactive oxygen species (ROS), such as the superoxide radical $\left(\mathrm{O}_{2}^{-}\right)$and hydrogen peroxide $\left(\mathrm{H}_{2} \mathrm{O}_{2}\right)$ (Liu et al., 2009; Borges et al., 2014). The accumulation of ROS damages lipids and proteins, results in cell death, and inhibits plant growth (Li et al., 2011). To alleviate ROS accumulation under stress conditions, both enzymatic and non-enzymatic antioxidants are present in plants. The enzymatic antioxidants include superoxide dismutase (SOD), catalase (CAT), glutathione reductase (GR), and ascorbate peroxidase (APX) (Asada, 1992), while the non-enzymatic antioxidants include glutathione (GSH) and ascorbate (ASA) (Li et al., 2011). The SOD catalyzes the dismutation of $\mathrm{O}_{2}$ to $\mathrm{O}_{2}$ and $\mathrm{H}_{2} \mathrm{O}_{2}$, which is subsequently reduced to $\mathrm{H}_{2} \mathrm{O}$ and $\mathrm{O}_{2}$ by CAT, APX, GR, etc. (Asada, 1992). ASA and GSH function as cofactors of enzymes of the antioxidant pathways, both can also directly quench ROS (Hernandez et al., 2001; Hossain et al., 2012).

The plant hormone abscisic acid (ABA) regulates many important plant developmental processes and is known to induce tolerance to various abiotic stresses; e.g., drought, salt, and low temperature, suggesting that it has significant agronomic potential (Giraudat et al., 1994). The involvement of ABA in mediating drought stress has been extensively explored, and many studies have examined the mechanism of ABA action at the physiological and molecular levels (Ferrandino and Lovisolo, 2014), and their findings have already been reviewed (Verslues and Zhu, 2007; Chinnusamy et al., 2008; Mehrotra et al., 2014). Increased levels of endogenous ABA have been reported in many plant species under abiotic stress, such as drought stress (Aimar et al., 2014), and exogenous ABA decreases ROS accumulation by inducing activities or expression levels of many antioxidative enzymes, resulting in enhanced abiotic tolerance, although the data supporting this hypothesis remain inconsistent. For instance, application of exogenous ABA in drought-stressed kiwifruit plant significantly enhances the activities of guaiacol peroxidase (POD), CAT, SOD, and APX (Wang et al., 2011). In drought-stressed Cotinus coggygia, however, ABA decreases activities of CAT, although it also markedly increases activities of SOD and POD (Li et al., 2010).

Moreover, exogenous ABA can increase content of ASA and GSH and enhance plant tolerance to abiotic stresses (Jiang and Zhang, 2002; Liu et al., 2011). To our knowledge, the molecular mechanism of ASA and GSH biosynthesis regulated by exogenous ABA application has not been reported. Wheat is an important drought-sensitive cereal crop whose growth and grain yield are severely affected by drought stress (Doyle and Fischer, 1979; Gao et al., 2011). Polyethylene glycol (PEG) 6000 is often used to stimulate drought stress in higher plants (Xiong et al., 2010; Benesova et al., 2012). In this study, transcript levels of the genes encoding ASA-GSH cycle enzymes were measured

Abbreviations: ABA, abscisic acid; ASA, ascorbate; DHAR, dehydroascorbate reductase; GPX, glutathione peroxidase; GR, glutathione reductase; GS, glutathione synthetase; GSH, glutathione; GST, glutathione-S-transferase; $\mathrm{H}_{2} \mathrm{O}_{2}$, hydrogen peroxide; MDA, malondialdehyde; MDHAR, monodehydroascorbate reductase; PEG, polyethylene-glycol; SA, salicylic acid. using qPCR in ABA-treated wheat seedlings suffering from PEGstimulated drought stress to help us further understand the molecular mechanism of ABA-enhanced drought tolerance in higher plants.

\section{Materials and Methods}

\section{Plant Materials and Growth Conditions}

Seeds of the common wheat (Triticum aestivum L.) cv. Yumai 34 were sterilized with $0.01 \%(\mathrm{~m} / \mathrm{v}) \mathrm{HgCl}_{2}$ followed by washing with distilled water. Sterilized seeds were grown hydroponically in full-strength Hoagland's solution (Elberse et al., 2003) in glass dishes (diameter $15 \mathrm{~cm}$ ) in a FPG-300C-30D incubator (Ningbo Laifu Technology Co., Beijing, China) under a 14-h photoperiod, irradiance of $250 \mu \mathrm{mol} \mathrm{m} \mathrm{m}^{-2} \mathrm{~s}^{-1}$, day/night temperature of $25 / 15^{\circ} \mathrm{C}$, and relative humidity of $60 / 75 \%$. Each dish contained approximately 60 seedlings. After 2 weeks seedlings showed approximately three leaves, and two dishes were grown under the above conditions with fresh Hoagland medium (control, $\mathrm{CK})$, two dishes were incubated with fresh Hoagland medium supplemented with PEG-6000 (15\%) solution for drought treatment, and another two dishes were transferred to Hoagland solution supplemented with PEG-6000 (15\%) plus $10 \mu \mathrm{M} \mathrm{ABA}$ (Wei et al., 2009) (PEG and ABA treatment, PEG + ABA). Solution $(100 \mathrm{~mL})$ was exchanged every day in all treatments. The uppermost fully expanded leaves and the longest roots of wheat seedlings were separately collected at $0,1,2,3,4$, and 5 days after initiating drought stress, and were immediately frozen in liquid nitrogen and stored at $-80^{\circ} \mathrm{C}$ before assessment of physiological parameters and gene expression profiles.

\section{Plant Measurements and Analysis}

Growth parameters (plant height, root length, fresh and dry weight of root and leaf tissues) were recorded every day during the stress period. Ten individual wheat seedlings were randomly harvested from each dish. Plant heights and dry and fresh weights of roots and leaves were measured and calculated.

\section{Assays of Malondialdehyde (MDA) and $\mathrm{H}_{2} \mathrm{O}_{2}$ Content in Leaves and Roots of Wheat Seedlings}

Lipid peroxidation was determined by estimating the MDA content using the method described by Zheng et al. (2008). The content of $\mathrm{H}_{2} \mathrm{O}_{2}$ was measured by monitoring the absorbance of the titanium-peroxidee complex at $390 \mathrm{~nm}$, following the method of Jessup et al. (1994).

\section{Assays of GSH and ASA Content}

The content of GSH and ASA was measured according to the methods of Kampfenkel et al. (1995) and Smith (1985), respectively.

\section{Determination of the Transcript Levels of the Eight Genes Encoding ASA-GSH Cycle Enzymes by qPCR}

The genes encoding ASA-GSH synthesis-related enzymes are illustrated in Supplementary Figure S1. These genes are glutathione-S-transferase 1 (GST1), glutathione-S-transferase 
2 (GST2), glutathione peroxidase 1 (GPX1), phospholipid hydroperoxide glutathione peroxidase 2 (GPX2), glutathione reductase $(G R)$, dehydroascorbate reductase (DHAR), monodehydroascorbate reductase (MDHAR), and glutathione synthetase (GS) (Chen et al., 2011), and the genes encoding these enzymes were previously isolated from common wheat in our laboratory (Li et al., 2013). Total RNA was extracted using the TRIzol reagent (Invitrogen, Carlsbad, CA, USA) according to the manufacturer's instructions and treated with RNase-free DNase I (Takara Biotechnology [Dalian] Co., Ltd., Dalian, China) to remove contaminating genomic DNA. First-strand cDNAs were synthesized from $2 \mu \mathrm{g}$ of total RNA using Super-Script II reverse transcriptase (Invitrogen, Carlsbad, CA, USA). qPCR was performed using a SYBR Premix Ex Taq (Perfect Real Time) kit (Takara Biotechnology [Dalian] Co., Ltd.) on a Light Cycler 480 Real-Time PCR System (Roche Diagnostics Ltd., West Sussex, UK) according to the manufacturer's instructions. Each reaction $(20 \mu \mathrm{L})$ comprised $10 \mu \mathrm{L}$ of SYBR Green Supermix $(2 \times), 1 \mu \mathrm{L}$ of diluted $\mathrm{cDNA}$, and $0.5 \mu \mathrm{L}$ of forward and reserve primers. The relative transcript levels were calculated using the $2^{-\Delta \Delta \mathrm{Ct}}$ method, with the wheat $\beta$-actin (GenBank Accession no. AB181991) and glyceraldehyde 3-phosphate dehydrogenase $(G A P D H)$ (GenBank Accession no. EF592180) genes as two internal controls. All primers are listed in Supplementary Table S1. Each data point was expressed as the average \pm SD of three independent replicates.

\section{Statistical Analysis}

Data were analyzed statistically using one-way analysis of variance and Duncan's multiple range tests to determine significant differences among group means. Significant differences from the control values were determined at $P<0.05$. All recorded values represent the means of the results of three replicates.

\section{Results}

\section{Effects of Exogenous ABA on Phenotypes and Growth Parameters of Wheat Seedlings Exposed to PEG-stimulated Drought-stress Conditions}

In the present study, wheat seedlings suffering from 15\% PEGstimulated drought stress showed time-dependent characteristics (Figure 1). Wheat seedlings suffering from drought stress showed no visible changes before 3 days, and then exhibited significant and deleterious phenotypes, such as curled and wilted leaves, shorter plant heights, and inhibited growth compared to control plants (Figure 1). These qualitative phenotypic effects were confirmed by quantitative analysis (Table 1). However, in the presence of exogenous $10 \mu \mathrm{M}$ ABA, the effect of drought stress on wheat seedling growth was significantly abrogated. This was also demonstrated by the significantly increased plant heights, fresh and dry shoot weights, and fresh and dry root weights (Table 1). After 5 days, plant height, shoot fresh weight, shoot dry weight, root fresh weight, and root dry weight of PEG + ABA treated wheat seedlings were significantly higher than those of sole PEG-treated wheat seedlings by $11.4,21.8,21.4,34.9$, and
$23.1 \%$, respectively. These results showed that ABA improved the drought tolerance of wheat seedlings.

In this study, we also found that growth of wheat seedlings was significantly inhibited by $10 \mu \mathrm{M}$ ABA under normal conditions, which was also confirmed by qualitative and quantitative data (Figure 1, Table 1, Supplementary Figure S2). We speculated that changes in transcriptional levels in this treatment may be associated with wheat growth, and not stress tolerance. In this study, wheat seedlings treated with $10 \mu \mathrm{M}$ ABA under normal conditions were not used in further experiments.

\section{MDA and $\mathrm{H}_{2} \mathrm{O}_{2}$ Content in Leaves and Roots of Wheat Seedlings Exposed to PEG-stimulated Drought Stress Conditions in Response to Exogenous ABA}

MDA and $\mathrm{H}_{2} \mathrm{O}_{2}$ content in drought and PEG + ABA treatments also increased in a time-dependent manner (Table 2). However, the MDA and $\mathrm{H}_{2} \mathrm{O}_{2}$ content in root and leaf tissues of PEG + ABA-treated wheat seedlings was significantly lower than those of solely PEG-treated wheat seedlings after 3 days of drought (Table 2). After 5 days of drought stress, the MDA and $\mathrm{H}_{2} \mathrm{O}_{2}$ content in root and leaf tissues of PEG + ABA-treated wheat seedlings were markedly lower than those of sole PEGtreated wheat seedlings by 8.7 and $31.0 \%$, and 13.3 and $22.6 \%$, respectively (Table 2 ). These results indicated that exogenous ABA application alleviated the accumulation of MDA and $\mathrm{H}_{2} \mathrm{O}_{2}$ induced by drought.

\section{Effects of Exogenous ABA on GSH and ASA Content in Root and Leaf Tissues of Wheat Seedlings Exposed to PEG-stimulated Drought Stress Conditions}

GSH content increased gradually in both leaf and root tissues of PEG-treated wheat seedlings after drought stress, and exogenous ABA application significantly accelerated this increase (Figures 2A,B). After 5 days of drought stress, GSH content in both root and leaf tissues of $\mathrm{PEG}+\mathrm{ABA}$ treated wheat seedlings was 29.9 and $33.3 \%$ higher, respectively, than those in the tissues of solely PEG-treated wheat seedlings. In contrast, ASA content in root and leaf tissues of solely PEG-treated wheat seedlings decreased rapidly with prolonged drought stress, but ABA application inhibited this effect (Figures 2C,D). After 5 days of drought stress, ASA content of the root and leaf of PEG + ABA-treated wheat seedlings was 68.5 and $49.7 \%$ higher, respectively, than those in the tissues of solely PEG-treated wheat seedlings.

\section{Transcript Levels of Genes Encoding Enzymes Involved in the ASA-GSH Cycle in Leaves and Roots of Wheat Seedlings Exposed to PEG-stimulated Drought Stress}

GST1, GST2, GPX1, GPX2, GR, DHAR, MDHAR, and GS transcript levels were measured using qPCR with the Actin gene as the internal control in leaf and root tissues of wheat seedlings (Figures 3, 4). Similar results were obtained using GAPDH 

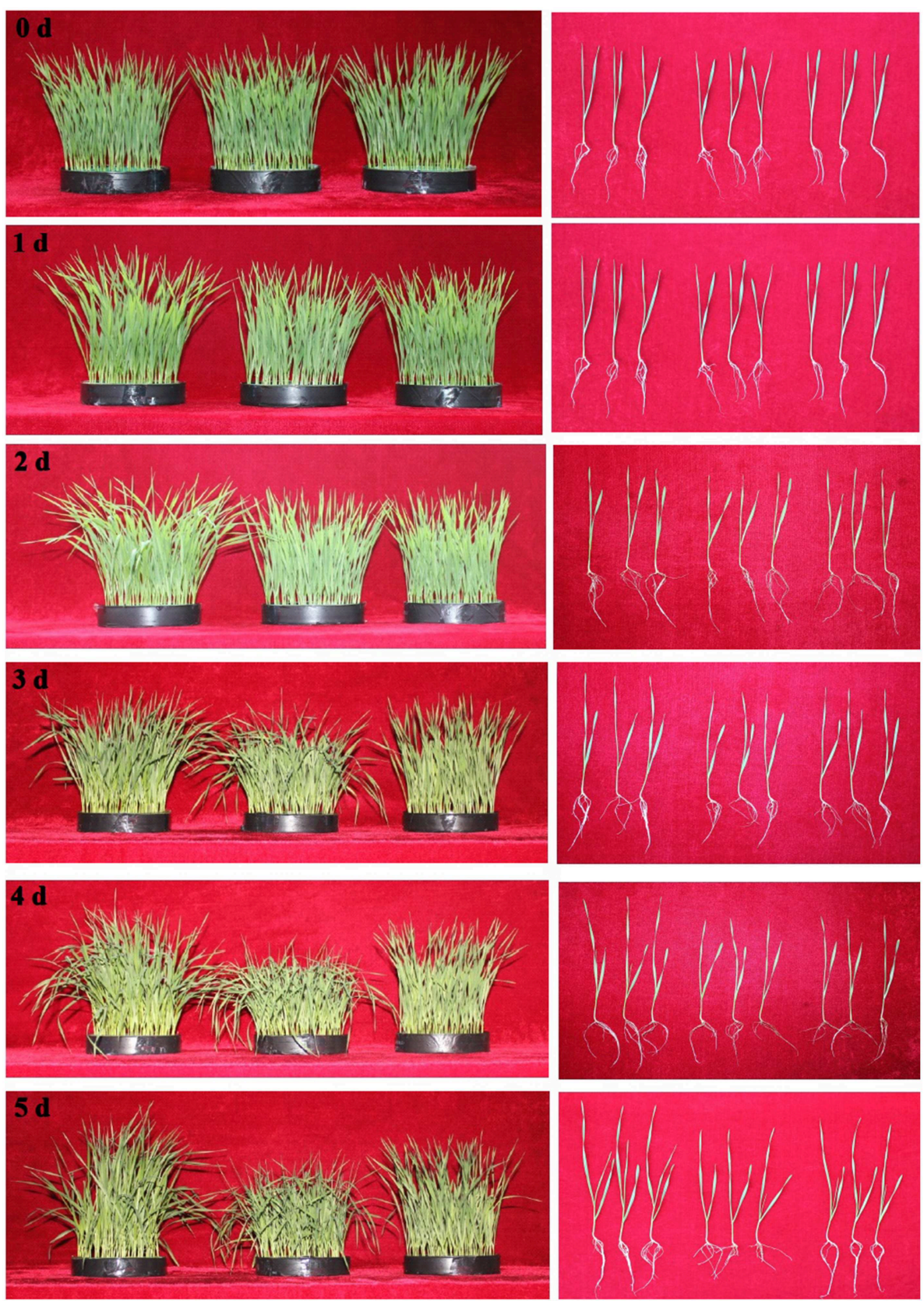

CK

PEG PEG + ABA

CK PEG PEG + ABA

FIGURE 1 | Phenotypic changes of wheat seedlings with exogenous application of $10 \mu \mathrm{M}$ ABA under $15 \%$ PEG-stimulated drought stress for 5 days. CK, control; PEG, 15\% PEG-6000; PEG + ABA, 15\% PEG and
$10 \mu \mathrm{M}$ ABA treatment. Three independent biological replications were performed with two dishes each, and about 60 wheat seedlings were planted in a dish. gene as another internal control, as indicated in Supplementary Figures S3, S4.

In root and leaf tissues of control wheat seedlings, the expression levels of the above mentioned eight genes encoding ASA-GSH synthesis-related enzymes remained almost constant (Figures 3, 4). Under PEG-stimulated drought-stress conditions, the expression patterns of these eight genes varied in the root and leaf tissues of wheat seedlings. In roots of PEG-treated wheat seedlings, GST1 expression was significantly induced at 1 day of stress, decreased rapidly, peaked again at 4 days, and then slowly decreased after 5 days of PEG-stimulated drought stress (Figure 3A). GST2, GPX1, GPX2, GR, MDHAR, and GS genes exhibited similar expression patterns in roots of wheat seedlings exposed to PEG-stimulated drought stress. Transcript 
TABLE 1 | Effect of exogenous ABA application on growth characteristics of wheat seedlings suffering from PEG-stimulated drought stress for 5 days.

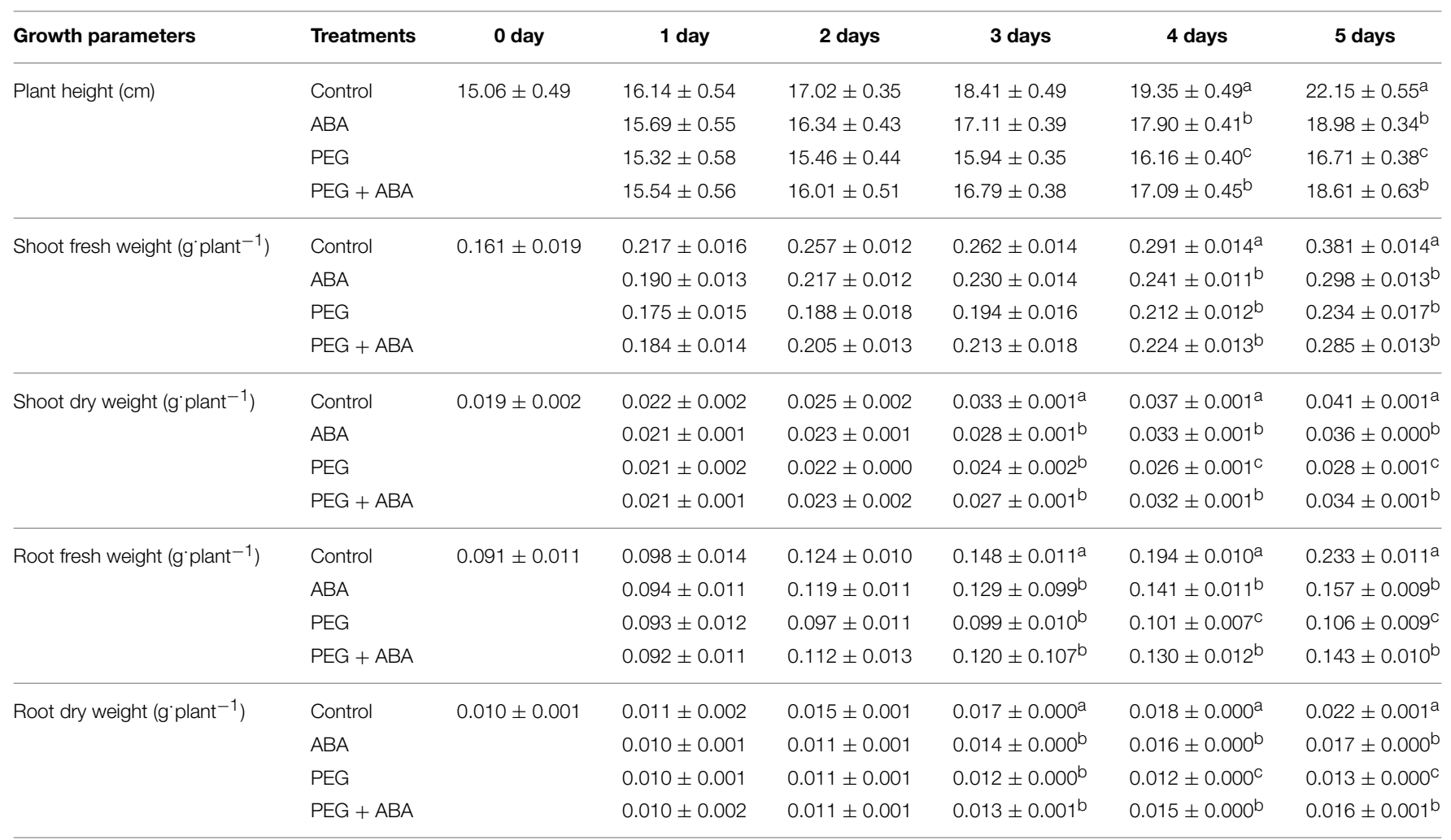

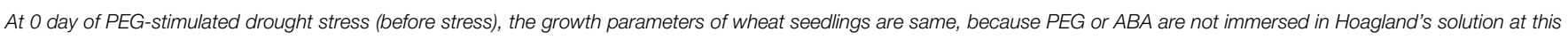

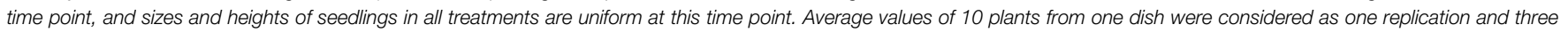
independent biological replications were performed. Different letters indicate a significant difference at $P<0.05$.

levels of these six genes were enhanced or inhibited in root tissue within 1-2 days after initiation of PEG-stimulated drought stress, peaked at 3 days, and slowly or rapidly decreased thereafter (Figures 3B-E,G,H). DHAR expression in roots was significantly induced at 1 day after PEG-stimulated drought stress, whereas it decreased rapidly thereafter (Figure 3F). During the PEGtreatment period, exogenous ABA significantly increased GST1 transcript levels at day 2 (Figure 3A), those of GPX1 at day 1 (Figure 3C), GPX2 at days 1 and 5 (Figure 3D), GR at days 1 and 2 (Figure 3E), DHAR at days 3, 4, and 5 (Figure 3F), and $M D H A R$ and $G S$ at days 1, 4, and 5 (Figures 3G,H) after PEG treatment.

During PEG-stimulated drought stress, transcript levels of eight genes encoding ASA-GSH synthesis-related enzymes in leaf differed significantly compared to those in root tissue. At all time points after PEG treatment, GST1 and GPX1 transcript levels were markedly inhibited (Figures 4A,C). GST2 and DHAR genes were induced early, peaked at 2 days after PEG treatment, and then rapidly decreased (Figures $4 \mathbf{B}, \mathbf{F}$ ). GPX2, GR, and GS expression levels were strongly inhibited at 1 day after PEG treatment, increased and peaked separately at days 2 and 3, then increased, and rapidly decreased at subsequent time points (Figures 4D,E,H). MDHAR transcript levels were enhanced at 1 day after PEG treatment, whereas they decreased rapidly at subsequent time points (Figure 4G). During the PEG-treatment period, exogenous ABA significantly increased the transcript levels of GST1 at days 1, 4, and 5 (Figure 4A), GST2 at days 3, 4, and 5 (Figure 4B), GPX1, GPX2 and GS at days 2, 3, and 4 (Figures 4C,D,H), GR at days 1, 3, and 4 (Figure 4E), DHAR at days 1 and 4 (Figure $4 \mathbf{F}$ ), and $M D H A R$ at days 1 and 3 (Figure 4G) after PEG treatment.

\section{Discussion}

\section{Exogenous ABA Enhances the Tolerance of Wheat Seedlings Suffering to PEG-stimulated Drought Stress}

Actual soil drought stress is rarely used, because components of soil are very complicated, and it is difficult to control all soil components. In addition, it is also very difficult to discriminate water stress from other abiotic stresses in soil system. However, it is important for water stress experiment to establish a stable and controlled condition (Zhang et al., 2004). PEG have been used extensively to induce plant water deficit in a relatively controlled manner, appropriate to experimental protocols because it is a very low chronic toxicity, molecules with mol wt greater than 3000 are apparently not absorbed at all, and plant water relations can be similar whether the plants are growing in soil or in a PEG solution having an equal water potential (Kaufmann and Eckard, 1971; Mexal et al., 1975; Carpita et al., 1979). 
TABLE 2 | Effect of exogenous ABA on content of MDA and $\mathrm{H}_{2} \mathrm{O}_{2}$ in root and leaf tissues of wheat seedlings suffering from PEG-stimulated drought stress for 5 days.

\begin{tabular}{|c|c|c|c|c|c|c|c|}
\hline Parameters & Treatments & 0 day & 1 day & 2 days & 3 days & 4 days & 5 days \\
\hline \multirow[t]{3}{*}{ MDA content in leaf } & Control & $5.06 \pm 0.10$ & $4.99 \pm 0.21$ & $4.95 \pm 0.17$ & $5.00 \pm 0.12$ & $5.09 \pm 0.14^{c}$ & $5.17 \pm 0.14^{C}$ \\
\hline & PEG & & $5.25 \pm 0.14$ & $5.83 \pm 0.13$ & $6.38 \pm 0.16$ & $6.66 \pm 0.13^{a}$ & $7.22 \pm 0.13^{a}$ \\
\hline & $\mathrm{PEG}+\mathrm{ABA}$ & & $5.16 \pm 0.16$ & $5.58 \pm 0.12$ & $5.91 \pm 0.12$ & $6.12 \pm 0.14^{b}$ & $6.59 \pm 0.14^{b}$ \\
\hline \multirow[t]{3}{*}{ MDA content in root } & Control & $5.59 \pm 0.31$ & $5.55 \pm 0.28$ & $5.52 \pm 0.24$ & $5.56 \pm 0.27^{c}$ & $5.45 \pm 0.25^{c}$ & $5.60 \pm 0.23^{C}$ \\
\hline & PEG & & $5.88 \pm 0.35$ & $6.40 \pm 0.27$ & $7.86 \pm 0.30^{a}$ & $9.11 \pm 0.28^{a}$ & $11.39 \pm 0.29^{a}$ \\
\hline & $\mathrm{PEG}+\mathrm{ABA}$ & & $5.75 \pm 0.28$ & $6.08 \pm 0.35$ & $6.39 \pm 0.36^{b}$ & $7.12 \pm 0.32^{b}$ & $7.86 \pm 0.20^{b}$ \\
\hline \multirow[t]{3}{*}{$\mathrm{H}_{2} \mathrm{O}_{2}$ content in leaf } & Control & $1.07 \pm 0.09$ & $1.04 \pm 0.07$ & $1.04 \pm 0.05$ & $1.08 \pm 0.07^{c}$ & $1.08 \pm 0.07^{C}$ & $1.02 \pm 0.07^{\mathrm{C}}$ \\
\hline & PEG & & $1.27 \pm 0.03$ & $1.42 \pm 0.10$ & $1.66 \pm 0.09^{a}$ & $1.88 \pm 0.08^{a}$ & $1.96 \pm 0.11^{\mathrm{a}}$ \\
\hline & $\mathrm{PEG}+\mathrm{ABA}$ & & $1.18 \pm 0.08$ & $1.26 \pm 0.06$ & $1.39 \pm 0.05^{b}$ & $1.55 \pm 0.08^{b}$ & $1.70 \pm 0.08^{b}$ \\
\hline \multirow[t]{3}{*}{$\mathrm{H}_{2} \mathrm{O}_{2}$ content in leaf } & Control & $1.19 \pm 0.07$ & $1.19 \pm 0.01$ & $1.19 \pm 0.08$ & $1.21 \pm 0.08^{\mathrm{C}}$ & $1.19 \pm 0.10^{C}$ & $1.22 \pm 0.07^{\mathrm{C}}$ \\
\hline & PEG & & $1.28 \pm 0.08$ & $1.46 \pm 0.05$ & $1.75 \pm 0.07^{a}$ & $2.31 \pm 0.07^{a}$ & $2.57 \pm 0.07^{a}$ \\
\hline & $\mathrm{PEG}+\mathrm{ABA}$ & & $1.23 \pm 0.05$ & $1.28 \pm 0.08$ & $1.44 \pm 0.05^{b}$ & $1.62 \pm 0.07^{b}$ & $1.99 \pm 0.06^{b}$ \\
\hline
\end{tabular}

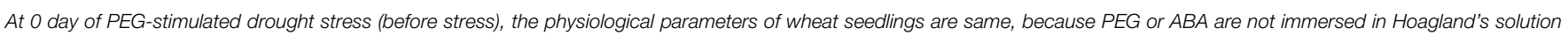

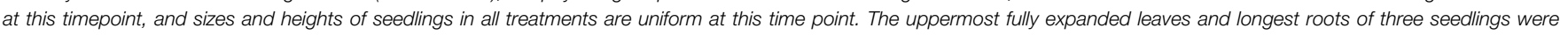

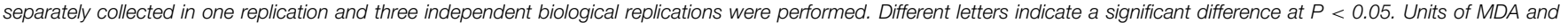
$\mathrm{H}_{2} \mathrm{O}_{2}$ content, umol.g $\mathrm{g}^{-1} \mathrm{FW}$.

In this study, application of exogenous $10 \mu \mathrm{M}$ ABA decreased the growth inhibition caused by $15 \%$ PEG 6000-stimulated drought stress, as manifested by increased growth parameters (plant height, shoot and root fresh weights, and shoot and root dry weights), and decreased $\mathrm{MDA}$ and $\mathrm{H}_{2} \mathrm{O}_{2}$ content (Tables 1, 2). These suggest that exogenous ABA enhances the tolerance of wheat seedlings to drought stress, similar to the previous reports in maize, bermudagrass, and grapemvine (Todorov et al., 1998; Lu et al., 2009; Ferrandino and Lovisolo, 2014). However, ABA-enhanced drought tolerance might also be related to its protective roles, such as closing stomata, and decreasing evapotranspiration and solute uptake (Kirkham, 1983). In addition, PEG has some disadvantages in stimulating water stress, including its uptake by plants, hypoxia, and mineral contamination (Lawlor, 1970; Janes, 1974; Reid, 1978; Yaniv and Werker, 1983; Jacomini et al., 1988; Verslues et al., 1998; Blum, 2013). Thus, ABA-induced drought tolerance in wheat plant could need to be further measured under actual soil drought stress conditions.

\section{ABA Temporally Regulates the Transcriptional Levels of the Genes Encoding ASA-GSH Cycle Enzymes in Wheat Seedlings, Resulting in Increased GSH and ASA Content}

GSH and ASA are major non-enzymatic antioxidants, and the enzymes and antioxidants in the ASA-GSH cycle play important roles in scavenging of ROS (Shan and Liang, 2010; Liu et al., 2012; Rakić et al., 2014). GSH and ASA content in abiotic-tolerant plant varieties are significantly higher than those in abiotic-sensitive varieties (Vaidyanathan et al., 2003). Overexpression of the genes encoding ASA-GSH cycle enzymes in higher plants confers enhanced tolerance to abiotic stresses (e.g., salt, low temperature) by maintaining higher content of GSH and ASA (Eltayeb et al.,
2006; Duan et al., 2012; Sultana et al., 2012). Our findings indicated that ASA content declined in solely PEG-treated wheat seedlings (Figure 2), indicating that PEG-stimulated drought stress could disturb synthesis of ASA. However, content of GSH increased in this treatment (Figure 2), possibly combating the oxidative stress generated due to drought stresses. This suggested that drought stress had the differential effects on between ASA and GSH synthesis. Under abiotic stresses, various expression profiles of different antioxidative enzymes and antioxidants have also been reported in previous studies ( $\mathrm{Li}$ et al., 2010; Wang et al., 2011; Hossain et al., 2012).

In the present study, much higher content of ASA and GSH was observed in both root and leaf tissues of PEG + ABA-treated wheat seedlings (Figure 2), implying that the drought tolerance enhanced by exogenous ABA application in wheat seedlings may be related to increased content of GSH and ASA. It has been reported that there may be no post-transcriptional, translational, or post-translational regulations of the genes encoding ASA-GSH cycle enzymes (Shan and Liang, 2010; Chen et al., 2011; Liu et al., 2012). Transcriptional analysis enables quantification of changes in transcript levels of genes. Therefore, transcriptional analysis can facilitate identification of genes involved in the regulation of metabolism and provide valuable insight into the molecular mechanisms of many biosynthetic pathways (Ohdan et al., 2005). In this study, the expression levels of eight genes encoding ASA-GSH cycle enzymes were determined in PEG-stimulated drought-stressed wheat seedlings to identify their associations with the increased GSH and ASA content of wheat seedlings after exogenous ABA application.

Our results showed that, in root and leaf tissues of PEGtreated wheat seedlings to which $\mathrm{ABA}$ had been applied, the transcript profiles of ASA-GSH synthesis-related genes varied in a time-dependent manner, and the transcript levels of at least 


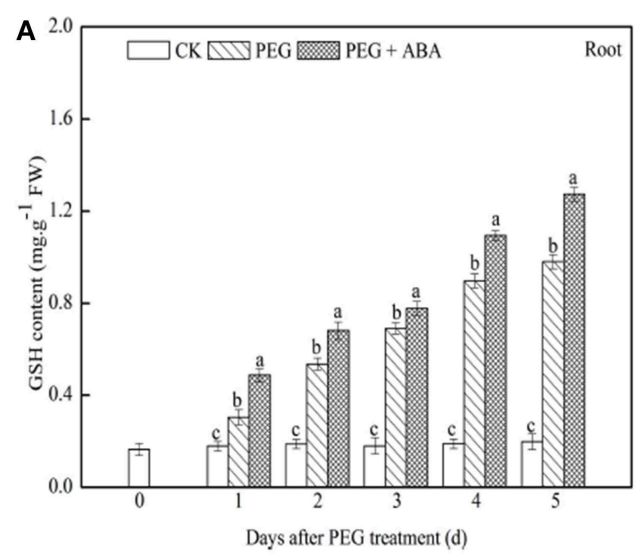

C

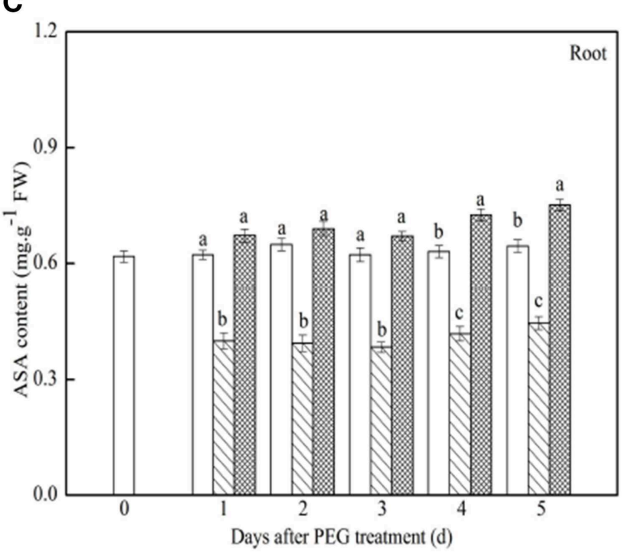

FIGURE 2 | Effects of exogenous ABA on content of GSH and ASA in roots and leaves of wheat seedlings suffered from PEG-stimulated drought stress. (A,C), GSH and ASA content in root of wheat seedling suffering from PEG-stimulated drought stress, respectively; (B,D), GSH and ASA content in leaf of wheat seedling

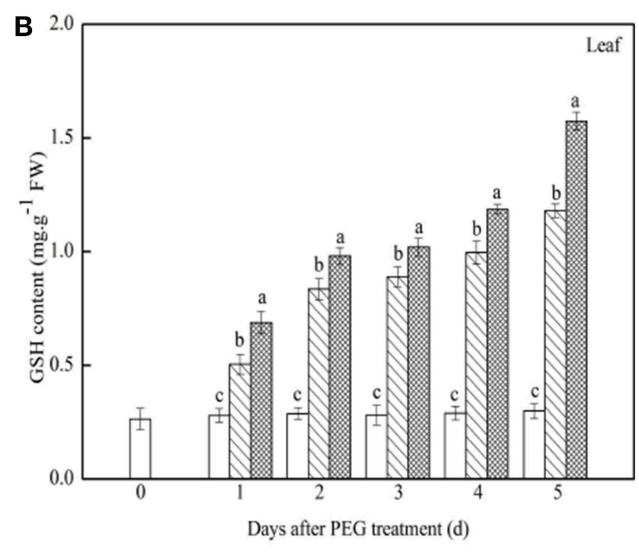

D

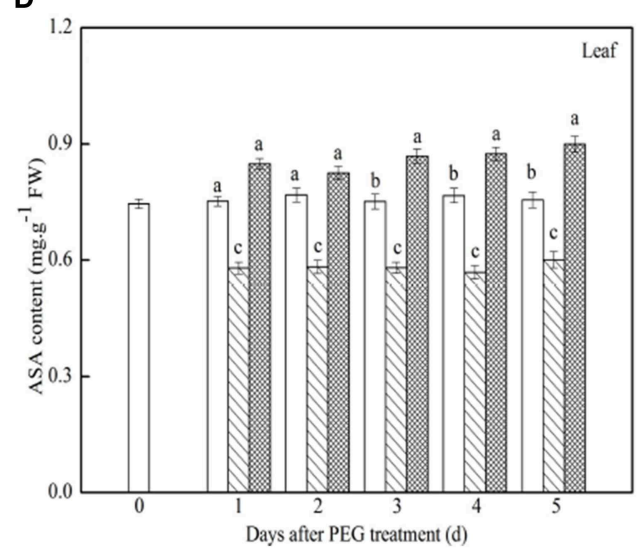

suffering from PEG-stimulated drought stress, respectively. The uppermost fully expanded leaves and longest roots of three seedlings were separately collected in one replication and three independent biological replications were performed. Different letters indicates significant differences $(P<0.05)$. one of the genes were markedly enhanced by ABA application at each time point (Figures 3, 4, Supplementary Figures S3, S4). This may be associated with the increased content of GSH and ASA during the PEG-stimulated drought-stress period in these two tissues, as well as the enhanced drought tolerance of PEG + ABA-treated wheat plants (Figure 1). These results are also supported by several previous studies of maize and wheat, in which the transcript levels of diverse genes encoding ASA-GSH cycle enzymes were temporally regulated by SA under cold- and salt-stress conditions (Liu et al., 2012; Li et al., 2013).

\section{Transcriptional Patterns of ASA-GSH Synthesis-related Genes in Root and Leaf Tissues of PEG-treated Wheat Seedlings Treated with Exogenous ABA}

Figures 3, 4 and Supplementary Figures S3, S4 show that the root and leaf tissues of drought-stressed wheat seedlings exhibited similar transcriptional patterns of ASA-GSH synthesis genes after pretreatment with ABA. Transcript levels of several genes at various time points, such as GR at day 1, DHAR at day 4, MDHAR at day 1 , and $G S$ at day 4 , were increased by $A B A$ in both the roots and leaves of PEG-treated wheat seedlings (Supplementary Table S2). These results suggested that root and leaf tissues may show similar responses to exogenous ABA application. However, differences in the transcriptional patterns in root and leaf tissues were also observed. For example, transcript levels of GST1 at days 1 and 4, GST2 at days 3, 4, and 5, GPX1 at days 2, 3, 4, GR at days 3 and 4, and $G S$ at days 2 and 3 significantly increased in the leaves of wheat seedlings subjected to PEG + ABA treatment, whereas transcript levels of these genes markedly decreased at the above time points in root tissue (Supplementary Table S2). These suggest that the mechanisms of action of ABA differ between root and leaf tissues of wheat seedlings. The differences in the transcript levels of ASA-GSH synthesis-related genes identified between root and leaf tissues may be related to differences in GSH and ASA content in the two tissues (Figure 2), or to the different functions, growth environments, and sensitivities of roots and leaves to PEG-stimulated drought stress and $\mathrm{ABA}$. The difference in the transcriptional profiles of root and leaf tissues further suggests that exogenous $\mathrm{ABA}$ application may have profound and distinct effects on these two tissues. 

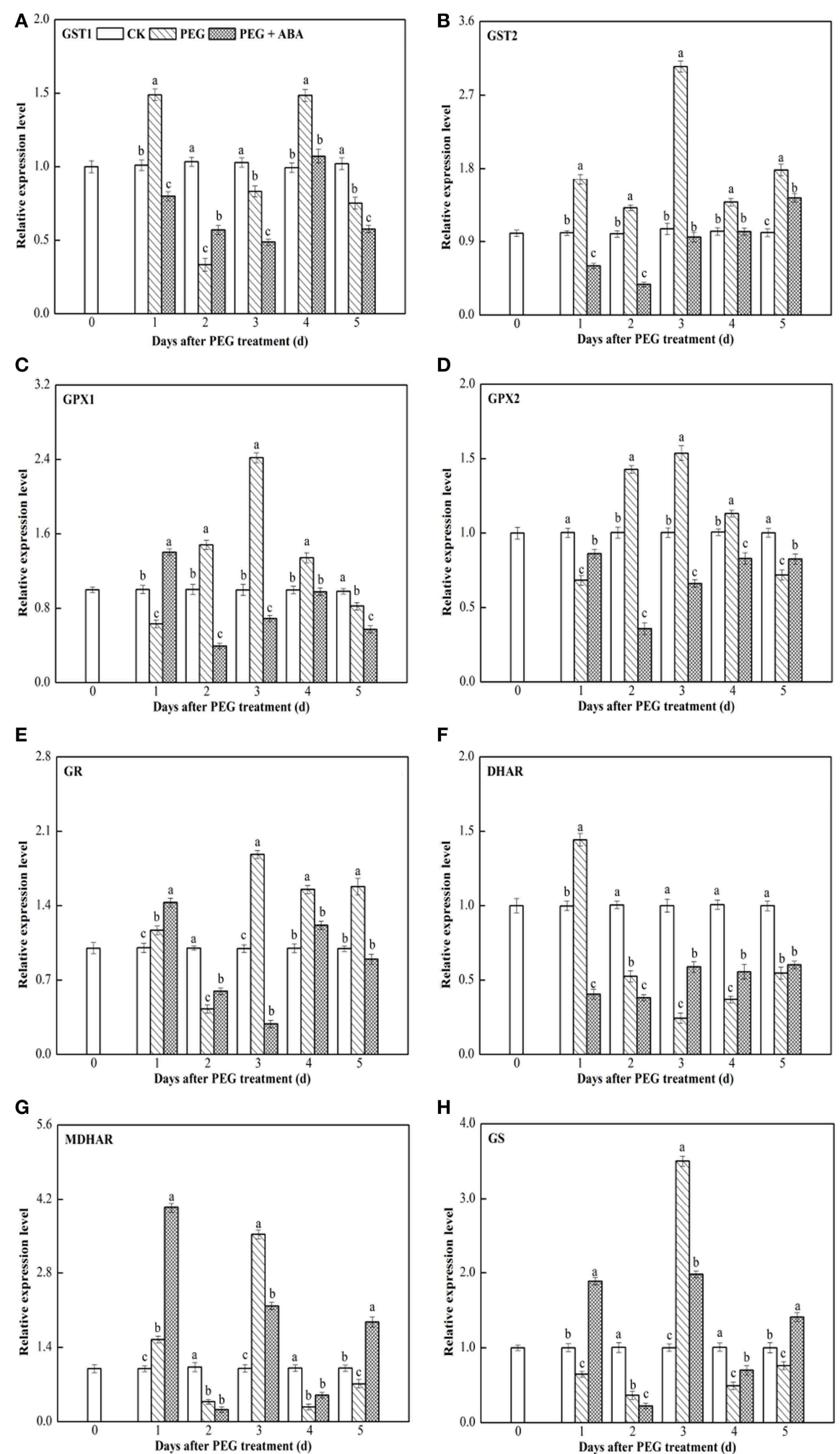

FIGURE 3 | Effects of exogenous ABA on transcript levels of the eight genes encoding ASA-GSH cycle enzymes in root of wheat seedlings suffering from PEG-stimulated drought stress.

Transcripts were analyzed by GPCR using Actin gene as internal control. (A-H), transcript levels of GST1, GST2, GPX1, GPX2, GR,

$D H A R, M D H A R$, and GS genes, respectively. The three seedlings were collected in one replication and three independent biological replications were performed. Each value is the mean \pm standard deviation of three independent measurements. Different letters indicate significant differences $(P<0.05)$. 

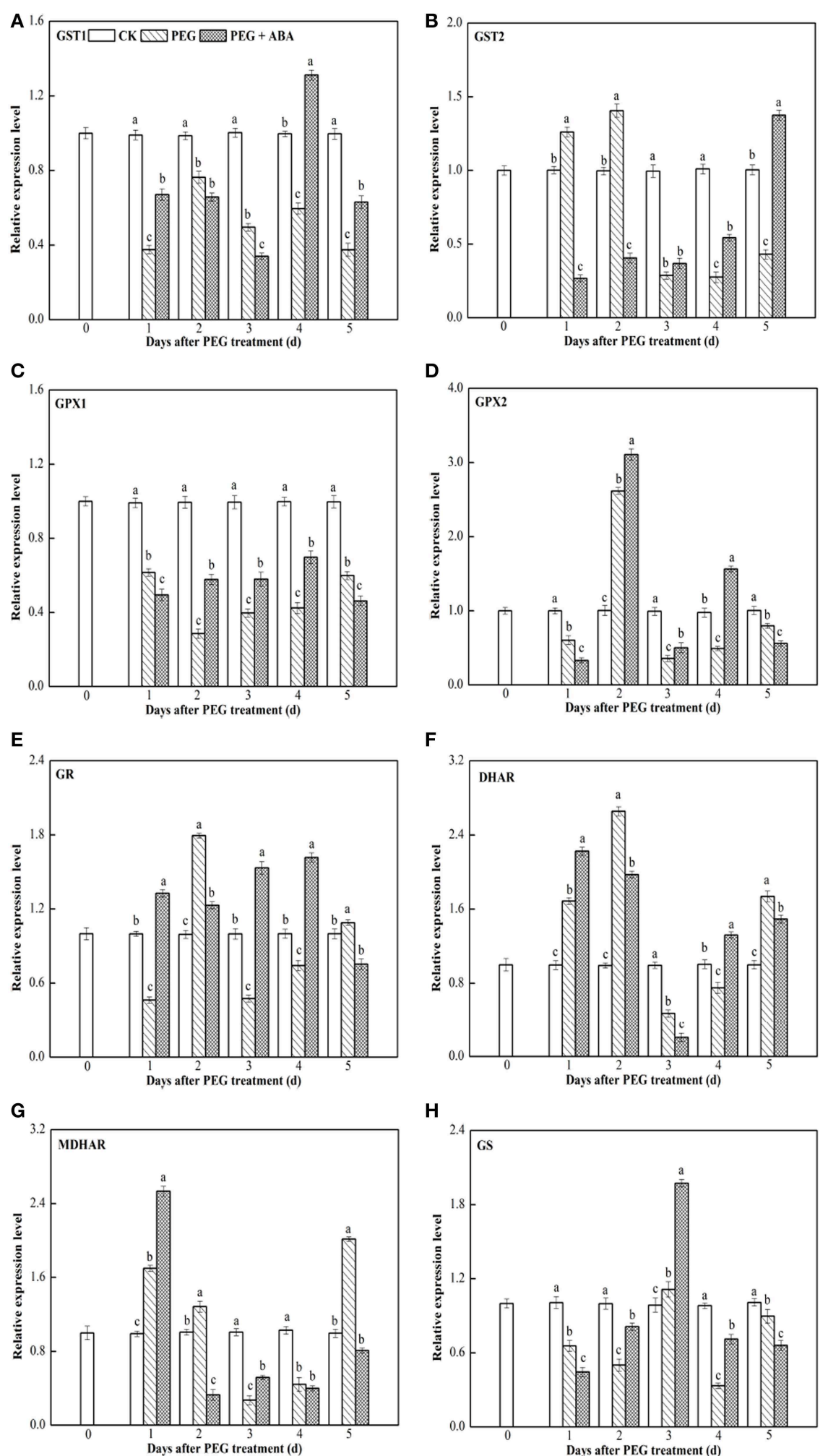

FIGURE 4 | Effects of exogenous ABA on transcript levels of the eight genes encoding ASA-GSH cycle enzymes in leaf of wheat seedlings suffering from PEG-stimulated drought stress. Transcripts were analyzed by qPCR using Actin gene as internal control. (A-H), transcript levels of GST1, GST2, GPX1, GPX2, GR, DHAR, MDHAR, and GS genes,

respectively. The uppermost fully expanded leaves and longest roots of three seedlings were collected in one replication and three independent biological replications were performed. Each value is the mean \pm standard deviation of three independent measurements. Different letters indicate significant differences $(P<0.05)$ 
Effects of ABA and Other Plant Hormones on the Transcriptional Profiles of Genes Encoding ASA-GSH Synthesis-related Enzymes under PEG-stimulated Drought Stress

qPCR has been used previously to examine the transcriptional profiles of genes encoding ASA-GSH synthesis-related enzymes regulated by SA in drought- and salt-stressed wheat seedlings, and cold-stressed eggplant (Chen et al., 2011; Kang et al., 2013; Li et al., 2013). The transcriptional profiles of genes encoding ASAGSH synthesis-related enzymes in the leaf tissue of PEG-treated wheat seedlings in the present study were compared to our previous findings on genes regulated by SA (Kang et al., 2013), because there are many similarities between the two studies; e.g., use of identical materials (leaf tissue of wheat seedlings), experimental conditions (15\% PEG-stimulated drought stress), and several sampling time-points ( 1 and 3 days after initiation of PEG-stimulated drought stress). Expression of GST at day 1, GST2 at day 3, GPX1 at day 2, GR at days 1 and 3, DHAR at days 2 and 3, MDHAR at day 1 , and $G S$ at day 2 were induced by both $\mathrm{ABA}$ and $\mathrm{SA}$ in the leaf tissue of wheat seedlings under PEGstimulated drought stress (Supplementary Table S3). However, the expression profiles of the majority of genes encoding ASAGSH synthesis enzymes were differentially regulated by ABA and SA under PEG-stimulated drought conditions (Supplementary Table S3). These results suggest that multiple plant hormones may increase the GSH and ASA content, possibly by differentially regulating the expression of genes encoding ASA-GSH synthesis enzymes.

\section{Conclusions}

Exogenous application of $10 \mu \mathrm{M}$ ABA significantly enhanced the tolerance of wheat seedlings to PEG-stimulated drought stress, as shown by alleviated growth inhibition, reduced content of MDA and $\mathrm{H}_{2} \mathrm{O}_{2}$, and increased content of GSH and ASA in the root and leaf tissues. The increased GSH and ASA content may

\section{References}

Aimar, D., Calafat, M., Andrade, A. M., Carassay, L., Bouteau, F., Abdala, G., et al. (2014). Drought effects on the early development stages of Panicum virgatum L.: cultivar differences. Biomass Bioenerg. 66, 49-59. doi: 10.1016/j.biombioe.2014.03.004

Asada, K. (1992). Ascorbate peroxidase - a hydrogen peroxide-scavenging enzyme in plants. Physiol. Plant. 85, 235-241. doi: 10.1111/j.1399-3054.1992.tb04728.x

Benesova, M., Hola, D., Fischer, L., Jedelsky, P. L., Hnilicka, F., Wilhelmova, N., et al. (2012). The physiology and proteomics of drought tolerance in maize: early stomatal closure as a cause of lower tolerance to short-term dehydration? PLoS ONE 7:e38017. doi: 10.1371/journal.pone.0038017

Blum, A. (2013). Use of PEG to Induce and Control Plant Water Deficit in Experimental Hydroponics' Culture. Available online at: www.plantstress.com/methods/peg

Borges, A. A., Jiménez-Arias, D., Expósito-Rodríguez, M., Sandalio, L. M., and Pérez, J. A. (2014). Priming crops against biotic and abiotic stresses: MSB as a tool for studying mechanisms. Front. Plant Sci. 5:642. doi: 10.3389/fpls.2014.00642

Carpita, N. C., Sabularse, D., Montezinos, D., and Delmer, D. P. (1979). Determination of the pore size of cell walls of living plant cells. Science 205, 1144-1147. doi: 10.1126/science.205.4411.1144 be associated with upregulated expression levels of ASA-GSH synthesis enzyme genes in time- and organ-specific manners. The transcriptional profiles of eight genes encoding ASA-GSH synthesis enzymes regulated by ABA differed between the root and leaf tissues of PEG-treated wheat seedlings. Comparison of our results with the findings of previous studies showed that, under PEG-stimulated drought-stress conditions, ABA and SA induced differential transcriptional profiles of genes encoding ASA-GSH synthesis enzymes in wheat seedlings. Our findings can provide specific information on the molecular mechanisms of the ASA and GSH synthesis regulated by ABA in droughtstressed plants.

\section{Author Contributions}

Liting Wei and Lina Wang performed the main experimental work, Liting Wei wrote the manuscript, Yang Yang and Pengfei Wang were responsible for viability tests and statistical analysis, Tiancai Guo designed the experiments, and Guozhang Kang provided the financial support and revised the manuscript.

\section{Acknowledgments}

This study was financially supported by the Program for Science and Technology Innovation Talents in Universities of Henan Province (15HASIT029), the Independent Item of the National Key Laboratory of Wheat and Maize Crop Science (SKL2014ZH03), and the Open Item of the State Key Laboratory of Crop Stress Biology for Arid Areas (CSBAA2015010).

\section{Supplementary Material}

The Supplementary Material for this article can be found online at: http://journal.frontiersin.org/article/10.3389/fpls.2015. 00458

Chen, S., Liu, Z., Cui, J., Ding, J., Xia, X., Liu, D., et al. (2011). Alleviation of chilling-induced oxidative damage by salicylic acid pretreatment and related gene expression in eggplant seedlings. Plant Growth Regul. 65, 101-108. doi: 10.1007/s10725-011-9579-9

Chinnusamy, V., Gong, Z., and Zhu, J. K. (2008). Abscisic acid-mediated epigenetic processes in plant development and stress responses. J. Integr. Plant Biol. 50, 1187-1195. doi: 10.1111/j.1744-7909.2008.00727.x

Doyle, A. D., and Fischer, R. A. (1979). Dry matter accumulation and water use relationships in wheat crops. Aust. J. Agr. Res. 30, 815-829. doi: 10.1071/AR9790815

Duan, M., Feng, H. L., Wang, L. Y., Li, D., and Meng, Q. W. (2012). Overexpression of thylakoidal ascorbate peroxidase shows enhanced resistance to chilling stress in tomato. J. Plant Physiol. 169, 867-877. doi: 10.1016/j.jplph.2012.02.012

Elberse, I. A. M., Van Damme, J. M. M., and Van Tienderen, H. P. (2003). Plasticity of growth characteristics in wild barley (Hordeum spontaneum) in response to nutrient limitation. J. Ecol. 91, 371-382. doi: 10.1046/j.1365-2745.2003.00776.x

Eltayeb, A. E., Kawano, N., Badawi, G. H., Kaminaka, H., Sanekata, T., Morishima, I., et al. (2006). Enhanced tolerance to ozone and drought stresses in transgenic tobacco overexpressing dehydroascorbate reductase in cytosol. Physiol. Plant. 127, 57-65. doi: 10.1111/j.1399-3054.2006.00624.x

Ferrandino, A., and Lovisolo, C. (2014). Abiotic stress effects on grapevine (Vitis vinifera L.): focus on abscisic acid-mediated consequences on secondary 
metabolism and berry quality. Environ. Exp. Bot. 103, 138-147. doi: 10.1016/j.envexpbot.2013.10.012

Gao, L., Yan, X., Li, X., Guo, G., Hu, Y., Ma, W., et al. (2011). Proteome analysis of wheat leaf under salt stress by two-dimensional difference gel electrophoresis (2D-DIGE). Phytochemistry 72, 1180-1191. doi: 10.1016/j.phytochem.2010.12.008

Giraudat, J., Parcy, F., Bertauche, N., Gosti, F., Leug, J., Morris, P. C., et al. (1994). Current advances in abscisic acid action and signaling. Plant Mol. Biol. 26, 1557-1577. doi: 10.1007/BF00016490

Hernandez, J. A., Ferrer, M. A., Jiménez, A., Barceló, A. R., and Sevilla, F. (2001). Antioxidant systems and $\mathrm{O}_{2}-/ \mathrm{H}_{2} \mathrm{O}_{2}$ production in the apoplast of pea leaves. Its relation with salt-induced necrotic lesions in minor veins. Plant Physiol. 127, 817-831. doi: 10.1104/pp.010188

Hossain, M. A., Piyatida, P., Teixeira da Silva, J. A., and Fujita, M. (2012). Molecular mechanism of heavy metal toxicity and tolerance in plants: central role of glutathione in detoxification of reactive oxygen species and methylglyoxal and in heavy metal chelation. J. Bot. 2012, 1-37. doi: $10.1155 / 2012 / 872875$

Jacomini, E., Bertani, A., and Mapelli, S. (1988). Accumulation of PEG 6000 and its effects on water content and carbohydrate level in water-stressed tomato plants. Can. J. Bot. 66, 970-973. doi: 10.1139/b88-140

Janes, B. E. (1974). The effect of molecular size, concentration in nutrient solution, and exposure time on the amount and distribution of polyethylene glycol in pepper plants. Plant Physiol. 54, 226-230. doi: 10.1104/pp.54.3.226

Jessup, W., Deean, R., and Gebicki, J. (1994). Iodometric determination of hydroperoxides in lipid and proteins. Methods Enzymol. 233, 289-298. doi: 10.1016/s0076-6879(94)33032-8

Jiang, M., and Zhang, J. (2002). Role of abscisic acid in water stress-induced antioxidant defense in leaves of maize seedlings. Free Radical Res. 36, 1001-1015. doi: 10.1080/1071576021000006563

Kampfenkel, K., Van Montaqu, M., and Inze, D. (1995). Extraction and determination of ascorbate and dehydroascorbate from plant tissue. Anal. Biochem. 225, 165-167. doi: 10.1006/abio.1995.1127

Kang, G. Z., Li, G. Z., Liu, G. Q., Xu, W., Peng, X. Q., Wang, C. Y., et al. (2013). Exogenous salicylic acid enhances drought tolerance and influences the expression patterns of ascorbate-glutathione biosynthesis-related genes in wheat seedlings. Biol. Plant. 57, 718-724. doi: 10.1007/s10535-013-0335-z

Kaufmann, M. R., and Eckard, A. N. (1971). Evaluation of water stress control with polyethylene glycols by analysis of guttation. Plant Physiol. 47, 453-456. doi: 10.1104/pp.47.4.453

Kirkham, M. B. (1983). Effect of ABA on the water relations of winter-wheat cultivars varying in drought resistance. Physiol. Plant. 59, 153-157. doi: 10.1111/j.1399-3054.1983.tb06587.x

Lawlor, D. W. (1970). Absorption of polyethylene glycols by plants and their effects on plant growth. New Phytol. 69, 501-513. doi: 10.1111/j.14698137.1970.tb02446.x

Li, G., Peng, X., Wei, L., and Kang, G. (2013). Salicylic acid increases the contents of glutathione and ascorbate and temporally regulates the related gene expression in salt-stressed wheat seedlings. Gene 529, 321-325. doi: 10.1016/j.gene.2013.07.093

Li, Q., Yu, B., Gao, Y., Dai, A. H., and Bai, J. G. (2011). Cinnamic acid pretreatment mitigates chilling stress of cucumber leaves through altering antioxidant enzyme activity. J. Plant Physiol. 168, 927-934. doi: 10.1016/j.jplph.2010.11.025

Li, Y., Zhao, H. X., Duan, B. L., Korpelainen, H., and Li, C. Y. (2010). Effect of drought and $\mathrm{ABA}$ on growth, photosynthesis and antioxidant system of Coinus coggygria seedlings under two different light conditions. Environ. Exp. Bot. 71, 107-113. doi: 10.1016/j.envexpbot.2010.11.005

Liu, Y., Jiang, H., Zhao, Z., and An, L. (2011). Abscisic acid is involved in brassinosteroids-induced chilling tolerance in the suspension cultured cells from chorispora bungeana. J. Plant Physiol. 168, 853-862. doi: 10.1016/j.jplph.2010.09.020

Liu, Y. J., Yuan, Y., Liu, Y. Y., Fu, J. J., Zheng, J., and Wang, G. Y. (2012). Gene families of maize glutathione-ascorbate redox cycle respond differently to abiotic stresses. J. Plant Physiol. 169, 183-192. doi: 10.1016/j.jplph.2011.08.018

Liu, Z. J., Zhang, X. L., Bai, J. G., Suo, B. X., Xu, P. L., and Wang, L. (2009). Exogenous paraquat changes antioxidant enzyme activities and lipid peroxidation in drought stressed cucumber leaves. Sci. Hortic. 121, 138-143. doi: 10.1016/j.scienta.2009.01.032

Lu, S., Su, W., Li, H., and Guo, Z. (2009). Abscisic acid improves drought tolerance of triploid bermudagrass and involves $\mathrm{H}_{2} \mathrm{O}_{2}$ - and NO-induced antioxidant enzyme activities. Plant Physiol. Biochem. 47, 132-138. doi: 10.1016/j.plaphy.2008.10.006

Mehrotra, R., Bhalothia, P., Bansal, P., Basantani, M. K., Bharti, V., and Mehrotra, S. (2014). Abscisic acid and abiotic stress tolerance-different tiers of regulation. J. Plant Physiol. 171, 486-496. doi: 10.1016/j.jplph.2013.12.007

Mexal, J., Fisher, J. T., Osteryoung, J., and Reid, C. P. P. (1975). Oxygen availability in polyethylene glycol solutions and its implication in plant-water relations. Plant Physiol. 55, 20-24. doi: 10.1104/pp.55.1.20

Ohdan, T., Francisco, P. B. Jr., Sawada, T., Hirose, T., Terao, T., Satoh, H., et al. (2005). Expression profiling of genes involved in starch synthesis in sink and source organs of rice. J. Exp. Bot. 56, 3229-3244. doi: 10.1093/jxb/eri292

Rakić, T., Lazarević, M., Jovanović, Ž. S., Radović, S., Siljak-Yakovlev, S., Stevanović, B., et al. (2014). Resurrection plants of the genus Ramonda: prospective survival strategies-unlock further capacity of adaptation, or embark on the path of evolution? Front. Plant Sci. 4:550. doi: 10.3389/fpls.2013.00550

Reid, C. P. (1978). Phosphorus contamination in polyethylene glycol. Plant Physiol. 61, 708-709. doi: 10.1104/pp.61.4.708

Shan, C. J., and Liang, Z. S. (2010). Jasmonic acid regulates ascorbate and glutathione metabolism in Agropyron cristatum leaves under water stress. Plant Sci. 178, 130-139. doi: 10.1016/j.plantsci.2009.11.002

Smith, I. K. (1985). Stimulation of glutathione synthesis in photorespiring plants by catalase inhibitors. Plant Physiol. 79, 1044-1047. doi: 10.1104/pp.79.4.1044

Sultana, S., Khew, C. Y., Morshed, M. M., Namasivayam, P., Napis, S., and Ho, C. L. (2012). Overexpression of monodehydroascorbate reductase from a mangrove plant (AeMDHAR) confers salt tolerance on rice. J. Plant Physiol. 169, 311-318. doi: 10.1016/j.jplph.2011.09.004

Todorov, D., Alexieva, V. V., and Karanov, E. (1998). Effect of putrescine, 4-PU-30, and abscisic acid on maize plants grown under normal, drought, and rewatering conditions. J. Plant Growth Regul. 17, 197-203. doi: 10.1007/PL00007035

Vaidyanathan, H., Sivakumar, P., Chakrabarty, R., and Thomas, C. (2003). Scavenging of reactive oxygen species in $\mathrm{NaCl}$-stressed rice (Oryza sativa $\mathrm{L}$.) differential response in salt-tolerant and sensitive varieties. Plant Sci. 165, 1411-1418. doi: 10.1016/j.plantsci.2003.08.005

Verslues, P. E., Ober, E. S., and Sharp, R. E. (1998). Root growth and oxygen relations at low water potentials. Impact of oxygen availability in polyethylene glycol solutions. Plant Physiol. 116, 1403-1412. doi: 10.1104/pp.116.4.1403

Verslues, P. E., and Zhu, J. K. (2007). New developments in abscisic acid perception and metabolism. Curr. Opin. Plant Biol. 10, 447-452. doi: 10.1016/j.pbi.2007.08.004

Wang, Y., Ma, F., Li, M., Liang, D., and Zou, J. (2011). Physiological responses of kiwifruit plants to exogenous ABA under drought conditions. Plant Growth Regul. 64, 63-74. doi: 10.1007/s10725-010-9537-y

Wei, K., Jin, X., Chen, X., Wu, F., Zhou, W., Qiu, B., et al. (2009). The effect of $\mathrm{H}_{2} \mathrm{O}_{2}$ and abscisic acid (ABA) interaction on beta-amylase activity under osmotic stress during grain development in barley. Plant Physiol. Biochem. 47, 778-784. doi: 10.1016/j.plaphy.2009.05.005

Xiong, J. H., Fu, B. Y., Xu, H. X., and Li, Y. S. (2010). Proteomic analysis of PEGsimulated drought stress-responsive proteins of rice leaves using a pyramiding rice line at the seedling stage. Bot. Stud. 51, 137-145.

Yaniv, Z., and Werker, E. (1983). Absorption and secretion of polyethylene glycol by Solanaceous plants. J. Exp. Bot. 34, 1577-1584. doi: 10.1093/jxb/34.11.1577

Zhang, L. J., Fan, J. J., Ruan, Y. Y., and Guan, X. Y. (2004). Application of polyethylene glycol in the study of plant osmotic stress physiology. Plant Physiol. 40, 361-364 (Chinese with English abstract).

Zheng, Y. H., Jia, A. J., Ning, T. Y., Xu, J. L., Li, Z. J., and Jiang, G. M. (2008). Potassium nitrate application alleviates sodium chloride stress in winter wheat cultivars differing in salt tolerance. J. Plant Physiol. 165, 1455-1465. doi: 10.1016/j.jplph.2008.01.001

Conflict of Interest Statement: The authors declare that the research was conducted in the absence of any commercial or financial relationships that could be construed as a potential conflict of interest.

Copyright (c) 2015 Wei, Wang, Yang, Wang, Guo and Kang. This is an open-access article distributed under the terms of the Creative Commons Attribution License (CC $B Y)$. The use, distribution or reproduction in other forums is permitted, provided the original author(s) or licensor are credited and that the original publication in this journal is cited, in accordance with accepted academic practice. No use, distribution or reproduction is permitted which does not comply with these terms. 